\title{
ORDERS IN SELF-INJECTIVE COGENERATOR RINGS
}

\author{
ROBERT C. SHOCK
}

\begin{abstract}
This note states necessary and sufficient conditions for a ring to be a right order in certain self-injective rings. A ring $R$ is said to have the dense extension property if each $R$-homomorphism from a right ideal of $R$ into $R$ can be lifted to some dense right ideal of $R$. A right ideal $K$ is rationally closed if for each $x \in R-K$ the set $x^{-1} K=\{y \in R: x y \in K\}$ is not a dense right ideal of $R$. We state a major result. Let $\operatorname{dim} R$ denote the Goldie dimension of a ring $R$ and $Z(R)$ the right singular ideal of $R$. Then $R$ is a right order in a self-injective cogenerator ring if and only if $R$ has the dense extension property, $Z(R)$ is rationally closed and the factor ring $R / Z(R)$ is semiprime with $\operatorname{dim} R / Z(R)=\operatorname{dim} R<\infty$.
\end{abstract}

1. Introduction. Let $R$ always denote a ring with 1. Recall, a ring is quasi-Frobenius if $R_{R}$ is right Artinian and $R_{R}$ is injective. For other equivalent definitions see [13] and for the original definition see [10]. Barbara Osofsky [13] has studied a generalization of the quasi-Frobenius ring, the self-injective cogenerator ring. A ring $R$ is a self-injective cogenerator ring if $R_{R}$ is injective and $R_{R}$ is a cogenerator in the category of unital right modules; this means that each right unital $R$-module can be embedded in a direct product of copies of $R$. The introduction of [13] gives a motivation for the study of these rings.

If $R$ is a subring of $Q$ and the identity of $R$ is also the identity of $Q$ then $R$ is a right order in $Q$ if

(a) every nonzero divisor of $R$ is a unit in $Q$, and

(b) every element of $Q$ can be written in the form of $c d^{-1}$ where $c$ and $d$ are in $R$ and $d$ is a nonzero divisor of $R$.

The problem of finding suitable conditions for a ring $R$ to be a right order in a quasi-Frobenius ring was first studied by Jans [7], then by Mewborn and Winton [12], Shock ([14], [15]), and Tachikawa [16]. These conditions require that $R$ be a solid Goldie ring [7], that is, $R$ satisfies the maximum condition on right annihilators of the injective hull of $R$. Proposition 8 replaces this solid Goldie condition by rings which satisfy

Received by the editors December 10, 1971.

AMS 1969 subject classifications. Primary 1625, 1650.

Key words and phrases. Injective, order, cogenerator, rationally closed, quasiFrobenius, dense right ideal, complete ring of right quotients.

c. American Mathematical Society 1972 
the minimum condition on annihilators of the injective hull of $R$. Our main result is an order theorem for self-injective cogenerator rings. The method of proof is via Theorem 5.3 of [15].

A right ideal $U$ of $R$ is called uniform if the intersection of any two nonzero right ideals of $R$ contained in $U$ is nonzero. If for some positive integer $n$ the ring $R$ contains an essential direct sum of $n$ uniform right ideals of $R$ then $R$ is called a right finite dimensional ring and we write $\operatorname{dim} R=n$. We denote the right singular ideal of $R$ by $Z(R)$, the complete ring of right quotients of $R$ by $Q\left[9\right.$, p. 94] and the injective hull of $R_{R}$ by $I(R)$. For $i \in I(R)$ and for a right ideal $H$ of $R$ we equate $i^{-1} H=\{x \in R: i x \in H\}$. A right ideal $L$ of $R$ is called dense if for $x \in R-0$ and $y \in R$ there is $r \in R$ such that $x r \neq 0$ and $y r \in L$; equivalently if $i \in I(R)$ and $i L=0$ then $i=0$ $[9$, p. 96]. Shock showed that a right ideal $K$ of $R$ is an annihilator of a subset of $I(R)$ if and only if $K$ is a rationally closed right ideal of $R$ [15]. For a general reference see [9].

In proving the main result which was stated in the abstract we first prove that a self-injective ring is a cogenerator if and only if the ring is right finite dimensional and the right singular ideal is rationally closed (Proposition 2). Finally, a ring $R$ is a right order in a quasi-Frobenius ring if and only if $R$ has the dense extension property and $R$ satisfies the minimum condition on rationally closed right ideals of $R$ and $Z(R)$ is the prime radical of $R$.

2. Self-injective cogenerator rings. We list a series of propositions which lead to our main theorem.

Proposition 1 (OSOFSKY [13]). A self-injective cogenerator ring is a right finite dimensional ring.

Proof. See Theorem 1 of [13].

It is clear that if $R$ contains no proper dense right ideals then every right ideal is rationally closed and conversely.

Proposition 2. Let $R$ be a self-injective ring. Then $R$ is a cogenerator if and only if $R$ is right finite dimensional and $Z(R)$ is rationally closed.

Proof. Since $R_{R}$ is injective, $R$ is a cogenerator if and only if each simple module is embedded in $R$ [13, Lemma 1]. Equivalently, if $M$ is a maximal right ideal of $R$ then $i M=0$ for some $i \in R-(0)$ which means that $R$ has no proper dense right ideals. If $R$ is a cogenerator then every right ideal is rationally closed and $R$ is finite dimensional by Proposition 1 . For the converse suppose that $D$ is a dense right ideal of $R$. Since $Z(R)$ is rationally closed, $(D+Z(R)) / Z(R)$ is dense in $R / Z(R)$ [15, Proposition 5.1]. However, $R / Z(R)$ is a semiprime Artinian ring because $R$ is a finite 
dimensional ring $[9, \mathrm{p} .103]$. Therefore, $R / Z(R)$ contains no proper dense right ideals and thus $D+Z(R)=R$. Since $Z(R)$ is the Jacobson radical of $R$ and is therefore a small submodule we have $D=R$. This completes the proof.

Proposition 3. The complete ring of right quotients $Q$ of $R$ is a selfinjective ring if and only if $R$ has the dense extension property.

Proof. This is an immediate consequence of the Findlay-LambekGabriel characterization of the complete ring of quotients as in [3], [4], or [6].

The most general condition for a ring to have a right classical quotient ring is the Ore condition [9, p. 109]. In working with theorems on orders there is always a method which in some sense replaces the Ore condition. We now state our method.

Proposition 4 (SHock [15]). Suppose that $Z(Q)$ is the Jacobson radical of $Q$ and is rationally closed. If $Q / Z(Q)$ is a semiprime Artinian ring and $R / Z(R)$ is semiprime then $R$ is a right order in $Q$.

Proof. We sketch a proof of Theorem 5.3 of [15]. Let $R / Z(Q)$ denote the embedded image of $R / Z(R)$ in $Q / Z(Q)$. Let $D$ be a dense right ideal of $R$. Since $Z(R)$ is rationally closed $(D+Z(Q)) / Z(Q)$ is dense in $Q / Z(Q)$. Hence $Q / Z(Q)$ is the complete ring of right quotients of $R / Z(Q)$ and $(D+Z(Q)) / Z(Q)$ contains an invertible element $d+Z(Q)$ in $Q / Z(Q)$. Also $d$ is invertible in $R$ since $Z(Q)$ is the Jacobson radical. A nonzero divisor $b$ of $R$ is a right nonzero divisor of $R / Z(Q)$ and $b+Z(Q)$ is invertible in $Q / Z(Q)$ and as before $b$ is invertible in $R$.

Recall $Q$ denotes the complete ring of right quotients of $R$. It is well known and easy to see that $\operatorname{dim} R=\operatorname{dim} Q$ whenever $R$ or $Q$ is a right finite dimensional ring.

THEOREM 5. A ring $R$ is a right order in a self-injective cogenerator ring if and only if

(I) $R$ has the dense extension property', and

(II) the right singular ideal $Z(R)$ is rationally closed and the factor ring $R / Z(R)$ is semiprime with $\operatorname{dim} R / Z(R)=\operatorname{dim} R<\infty$.

Proof. Assume that $R$ is a right order in $Q$ and $Q$ is a self-injective cogenerator ring. Then $Q$ has no proper dense right ideals and $Z(Q)$ is rationally closed. Since $Z(Q) \cap R=Z(R), Z(R)$ is rationally closed. Since $Q$ is a classical ring of right quotients of $R$ it follows that $Q / Z(Q)$ is semiprime Artinian and is a classical ring of right quotients of $R / Z(R)$. Hence $R / Z(R)$ is semiprime and $\operatorname{dim} R / Z(R)=\operatorname{dim} Q / Z(Q)$. Also, $\operatorname{dim} R=\operatorname{dim} Q$ and finally $\operatorname{dim} Q=\operatorname{dim} Q / Z(Q)$ because idempotents may be lifted modulo 
$Z(Q)[9, \mathrm{p} .103]$ and primitive idempotents remain primitive $[9, \mathrm{p} .75]$. Therefore, $\operatorname{dim} R=\operatorname{dim} R / Z(R)$. For the converse let $Q$ denote the complete ring of right quotients of $R$. By (I) and by Proposition 3 the ring $Q$ is self-injective. Also, $\operatorname{dim} Q=\operatorname{dim} R$ and $Q$ is semiperfect [9, p. 103]. Therefore, $\operatorname{dim} Q=\operatorname{dim} Q / Z(Q)$ because primitive idempotents remain primitive modulo $Z(Q)$. If $Z(Q)$ is rationally closed then by Proposition 4 the ring $R$ is a right order in $Q$. Furthermore the proof of Theorem 5.3 of [15] (or the proof of Proposition 2) shows that $Q$ has no proper dense right ideals. Thus, $Q$ is a self-injective cogenerator ring. It suffices to show that $Z(Q)=H$ where $H=r(l(Z(Q)))$ and $l(Z(Q))=\{x \in Q: x Z(Q)=0\}$ and $r(l(Z(Q)))=\{y \in Q: l(Z(Q)) y=0\}$. Clearly, $H \supseteq Z(Q)$ and if $x \in Q$ then $x Z(R)=0$ if and only if $x Z(Q)=0$. Also, $H$ is an ideal since $Z(Q)$ is. Hence, $H=r(l(Z(R)))$. If $y \in R-Z(R)$ there is $i \in I(R)=Q$ such that $i Z(R)=0$ and $i y \neq 0$. We conclude that $y \in R-H$ and $H \cap R=Z(R)$. This means that $R / Z(R)$ is embedded in $Q / H$ and by Lemma 5.2 of [15], $Q / H$ is a ring of right quotients of the embedded ring of $R / Z(R)$. Therefore, $\operatorname{dim} Q / H=$ $\operatorname{dim} R / Z(R)$. Recall $\operatorname{dim} Q / Z(Q)=\operatorname{dim} Q=\operatorname{dim} R$. By (II), $\operatorname{dim} R=$ $\operatorname{dim} R / Z(R)$ and we have $\operatorname{dim} Q / H=\operatorname{dim} Q / Z(Q)$. Therefore, $H=Z(Q)$ because $Q / Z(Q)$ is a semiprime Artinian ring and $H \supseteq Z(Q)$.

An ideal $L$ of $R$ is left $T$-nilpotent if for each sequence $x_{1}, x_{2}, \cdots$ of $L$ there is a corresponding integer $n$ such that $x_{1} x_{2} \cdots x_{n}=0$. Assume that $R$ modulo the Jacobson radical $J$ of $R$ is a semiprime Artinian ring. Then $R$ is left perfect if $J$ is left $T$-nilpotent. Also $R$ is a semiprimary ring if $J$ is nilpotent.

COROLlaRy 6. A ring $R$ is a right order in a self-injective cogenerator ring which is left perfect (semiprimary) if and only if the prime radical of $R$ is left T-nilpotent (nilpotent) and (I) and (II) of Theorem 5 hold.

Proof. Suppose $R$ is a right order in $Q$. If $a_{i} b_{i}^{-1} \in Z(Q)$ for $1 \leqq i \leqq n$ then $a_{1} b_{1}^{-1} \cdots a_{n} b_{n}^{-1}=a_{1} x_{2} \cdots x_{n} a^{-1}$ where $x_{i} \in Z(R)$ and $b_{i}^{-1} a_{i+1}=$ $x_{i+1} c_{i}^{-1}$ for appropriate $c_{i} \in R, 1 \leqq i \leqq n-1$. The proof is straightforward and details are omitted.

LEMMA 7. If $R$ has the minimum condition on rationally closed right ideals then so does $Q$ and conversely.

Proof. Suppose $K$ and $H$ are two rationally closed right ideals of $Q$ with $K \supsetneqq H$. There is $i \in I(R)$ such that $i H=0$ and $i k \neq 0$ for some $k \in K$. Since $k \in Q, k^{-1} R$ is dense $\left[9\right.$, p. 96]. Thus, $(i k)\left(k^{-1} R\right) \neq 0$ and $i(K \cap R) \neq 0$ implies that $(K \cap R) \supsetneqq(H \cap R)$. Also $K \cap R$ and $H \cap R$ are rationally closed in $R$ and the result follows. 
PROPOSITION 8. A ring $R$ is a right order in a quasi-Frobenius ring if and only if

(1) $R$ has the dense extension property,

(2) $R$ satisfies the minimum condition on rationally closed right ideals of $R$, and

(3) the prime radical of $R$ is the right singular ideal of $R$.

Proof. Suppose $R$ is a right order in a quasi-Frobenius ring $Q$. Proposition 3 implies (1) and Lemma 7 implies (2). Since $R$ is a right order in $Q, R / Z(R)$ is a right order in $Q / Z(Q)$ and hence $R / Z(R)$ is semiprime. Also $Z(R)$ is nilpotent since $Z(Q)$ is and (3) follows. For the converse, (1) and (2) imply that $Q$ is a self-injective ring with the minimum condition on right annihilators. This implies the maximum condition on left annihilators of the ring $Q$. Since $Q_{Q}$ is injective, $l(r(L))=L$ where $r(L)=\{x \in Q: L x=0\}$, $l(r(L))=\{y \in Q: y(r(L))=0\}$ for every finitely generated left ideal $L$ of $Q$. The collection of finitely generated left ideals of $Q$ satisfies the maximum condition and ${ }_{Q} Q$ is left Noetherian. Therefore, $Q$ is quasi-Frobenius and $Q$ with (3) above satisfies the hypothesis of Proposition 4 . Therefore, $R$ is a right order in $Q$.

AdDED IN PROOF. Morita [Sci. Rep. Tokyo Kyoiku Daigaku Sect. A 6 (1958), 83-142] introduced injective cogenerator rings and showed that the Artinian ones were quasi-Frobenius. Walker and Faith [J. Algebra 5 (1967), 203-221] introduced cogenerator rings and showed that the semilocal ones were self-injective.

\section{REFERENCES}

1. H. Bass, Finistic dimension and a homological generalization of semi-primary rings, Trans. Amer. Math. Soc. 95 (1960), 466-488. MR 28 \#1212.

2. C. Faith, Lectures on injective modules and quotient rings, Lecture Notes in Math., no. 49, Springer-Verlag, Berlin and New York, 1967. MR 37 \#2791.

3. G. D. Findlay and J. Lambek, A generalized ring of quotients. I, II, Canad. Math. Bull. 1 (1958), 77-85; 155-167. MR 20 \#888.

4. G. Gabriel, Des catégories abeliennes, Bull. Soc. Math. France 90 (1962), 323 448.

5. A. W. Goldie, Semi-prime rings with the maximum condition, Proc. London Math. Soc. (3) 10 (1960), 201-220. MR 22 \#2627.

6. O. Goldman, Rings and modules of quotients, J. Algebra 13 (1969), 10--47. MR 39 \#6914.

7. J. P. Jans, On orders in quasi-Frobenius rings, J. Algebra 7 (1967), 35 43. MR 35 \#2924.

8. R. E. Johnson, The extended centralizer of a ring over a module, Proc. Amer. Math. Soc. 2 (1951), 891-895. MR 13, 618.

9. J. Lambek, Lectures on rings and modules, Blaisdell, Waltham, Mass. 1966. MR 34 \#5857.

10. T. Nakayama, On Frobeniusean algebras. I, Ann. of Math. (2) 40 (1939), 611-633 MR 1, 3. 
11. T. Nakayama, On Frobeniusean algebras. II, Ann. of Math. (2) 42 (1941), 1-21. MR 2, 344.

12. A. C. Mewborn and C. N. Winton, Orders in self-injective semi-perfect rings, J. Algebra 13 (1969), 5-9. MR 39 \#6925.

13. B. L. Osofsky, A generalization of quasi-Frobenius rings, J. Algebra 4 (1966), 373387; Erratum, ibid. 9 (1968), 120. MR 34 \#4305; MR 36 \#6443.

14. R. C. Shock, Right orders in self-injective rings, Notices Amer. Math. Soc. 17 (1970), 561. Abstract \#70T-A76.

15. —- Injectivity, annihilators, and orders, J. Algebra 19 (1971), 96-103.

16. H. Tachikawa, Localization and Artinian quotient rings, Math. Z. 119 (1971), 239253.

Department of Mathematics, Southern Illinois University, Carbondale, IlliNoIS 62901 